\title{
Article \\ Starlikeness of meromorphic functions involving certain differential inequalities
}

\author{
Kuldeep Kaur Shergill ${ }^{1, *}$ and Sukhwinder Singh Billing ${ }^{1}$ \\ 1 Department of Mathematics, Sri Guru Granth Sahib World University, Fatehgarh Sahib-140407(Punjab), India. \\ * Correspondence: kkshergill16@gmail.com \\ Communicated by: Absar ul Haq \\ Received: 27 October 2020; Accepted: 31 March 2021; Published: 21 May 2021.
}

Abstract: In the present paper, we define a class of non-Bazilevic functions in punctured unit disk and study a differential inequality to obtain certain new criteria for starlikeness of meromorphic functions.

Keywords: Meromorphic function; Meromorphic starlike function.

MSC: 30C45; 30C80.

\section{Introduction}

$\mathbf{L}$

et $\Sigma$ be the class of functions of the form

$$
f(z)=\frac{1}{z}+\sum_{0}^{\infty} a_{n} z^{n}
$$

which are analytic in the punctured unit disc $\mathbb{E}_{0}=\mathbb{E} \backslash\{0\}$, where $\mathbb{E}=\{z:|z|<1\}$. A function $f \in \Sigma$ is said to be meromorphic starlike of order $\alpha$ if $f(z) \neq 0$ for $z \in \mathbb{E}_{0}$ and

$$
-\Re\left(\frac{z f^{\prime}(z)}{f(z)}\right)>\alpha, \quad(\alpha<1 ; z \in \mathbb{E}) .
$$

The class of such functions is denoted by $\mathcal{M S}^{*}(\alpha)$ and the class of meromorphic starlike functions is denoted by $\mathcal{M S}^{*}=\mathcal{M S}^{*}(0)$.

In the theory of meromorphic functions, many authors have obtained different sufficient conditions for meromorphically starlike functions. Some of them are stated below:

Kargar et al., [1] proved the following results:

Theorem 1. Assume that $f(z) \neq 0$ for $\mathbb{E}_{0}$. If $f \in \Sigma(p)$ satisfies

$$
\left|\frac{1}{\sqrt[p]{f(z)}}\left(\frac{f^{\prime}(z)}{f(z)}+\right)+p\right|<p \lambda(\beta)|b(z)|, z \in \mathbb{E}_{0},
$$

then $f$ is a p-valently meromorphic strongly-starlike of order $\beta$.

Theorem 2. Assume that $f(z) \neq 0$ for $\mathbb{E}_{0}$. If $f \in \Sigma$ satisfies

$$
\left|\left(\frac{f(z)}{z^{-\alpha}}\right)^{\frac{1}{\alpha-1}}\left(\frac{f^{\prime}(z)}{f(z)}+\frac{\alpha}{z}\right)+1-\alpha\right|<\frac{2}{\sqrt{5}}, z \in \mathbb{E}_{0},
$$

then $f$ is meromorphic starlike function of order $\alpha$.

Goswami et al., [2] proved the following results: 
Theorem 3. If $f(z) \in \Sigma_{p}, n$ with $f(z) \neq 0$ for all $z \in \mathbb{E}_{0}$, satisfies the following inequality

$$
\left|\left[z^{p} f(z)\right]^{\frac{1}{\alpha-p}}\left(\frac{z f^{\prime}(z)}{f(z)}+\alpha\right)+p-\alpha\right|<\frac{(n+1)(p-\alpha)}{\sqrt{(n+1)^{2}+1}}, z \in \mathbb{E},
$$

for some real values of $\alpha(0 \leq \alpha<p)$, then $f \in \mathcal{M S}_{p, n}^{*}(\alpha)$.

Theorem 4. If $f(z) \in \Sigma_{p}, n$ with $f(z) \neq 0$ for all $z \in \mathbb{E}_{0}$ satisfies the following inequality

$$
\left|\frac{\gamma\left[z^{p} f(z)\right]^{\gamma}}{z}\left(\frac{z f^{\prime}(z)}{f(z)}+p\right)\right| \leq \frac{(n+1)}{2 \sqrt{(n+1)^{2}+1}}, z \in \mathbb{E},
$$

for $\gamma \leq-\frac{1}{p}$, then $f \in \mathcal{M S}_{p, n}^{*}\left(p+\frac{1}{\gamma}\right)$.

In [3], Sahoo et al., investigated a new class $\mathcal{U}_{n}(\alpha, \lambda, \mu)$, of non-Bazilevic analytic functions by

$$
\mathcal{U}_{n}(\alpha, \lambda, \mu)=\left\{f \in \mathcal{A}_{n}:\left|(1-\alpha)\left(\frac{z}{f(z)}\right)^{\mu}+\alpha f^{\prime}(z)\left(\frac{z}{f(z)}\right)^{\mu+1}-1\right|<\lambda, z \in \mathbb{E}\right\} .
$$

For different choices of $\mu$ with $\alpha=1$, many authors has studied this class which are included in [4-6]. In this paper, we define above class of non-Bazilevic functions in punctured unit disk and study a differential inequality to obtain certain new criteria for starlikeness of meromorphic functions.

\section{Main results}

To prove our main result, we shall make use of following lemma of Hallenback and Ruscheweyh [7].

Lemma 1. Let $G$ be a convex function in $\mathbb{E}$, with $G(0)=a$ and let $\gamma$ be a complex number, with $\Re(\gamma)>0$. If $F(z)=a+a_{n} z^{n}+a_{n+1} z^{n+1}+\ldots$, is analytic in $\mathbb{E} n d F \prec G$, then

$$
\frac{1}{z^{\gamma}} \int_{0}^{z} F(w) w^{\gamma-1} d w \prec \frac{1}{n z^{\frac{\gamma}{n}}} \int_{0}^{z} G(w) w^{\frac{\gamma}{n}-1} d w .
$$

Theorem 5. Let $\alpha, \beta, \delta$ be real numbers such that $\alpha<\frac{2}{\delta-1}, \beta>0,0 \leq \delta<1$ and let

$$
0<M \equiv M(\alpha, \beta, \delta)=\frac{(\beta-\alpha)[2+\alpha(1-\delta)]}{\alpha[1+\beta(1-\delta)]} .
$$

If $f \in \Sigma$ satisfies the differential inequality

$$
\left|\left(\frac{1}{z f(z)}\right)^{\beta}\left(1+\alpha+\alpha \frac{z f^{\prime}(z)}{f(z)}\right)-1\right|<M(\alpha, \beta, \delta), z \in \mathbb{E},
$$

then

$$
-\Re\left(\frac{z f^{\prime}(z)}{f(z)}\right)>\delta, z \in \mathbb{E}
$$

Proof. Let us define

$$
\left(\frac{1}{z f(z)}\right)^{\beta}=u(z), z \in \mathbb{E}
$$


Differentiate logarithmically, we obtain

$$
\frac{z f^{\prime}(z)}{f(z)}=-\left(1+\frac{z u^{\prime}(z)}{\beta u(z)}\right)
$$

Therefore, in view of (3), we have

$$
u(z)-\frac{\alpha}{\beta} z u^{\prime}(z) \prec 1+M z .
$$

The use of Lemma $1\left(\right.$ taking $\left.\gamma=-\frac{\beta}{\alpha}\right)$ in (4) gives

$$
u(z) \prec 1+\frac{\gamma M z}{\gamma+1}
$$

or

$$
|u(z)-1|<\frac{\beta M}{\beta-\alpha}<1,
$$

therefore, we obtain

$$
|u(z)|>1-\frac{\beta M}{\beta-\alpha}
$$

Write $-\frac{z f^{\prime}(z)}{f(z)}=(1-\delta) w(z)+\delta, 0 \leq \delta<1$ and therefore (2) reduces to

$$
|(1+\alpha) u(z)-\alpha u(z)[(1-\delta) w(z)+\delta]-1|<M .
$$

We need to show that $\Re(w(z))>0, z \in \mathbb{E}$. If possible, suppose that $\Re(w(z)) \ngtr 0, z \in \mathbb{E}$, then there must exist a point $z_{0} \in \mathbb{E}$ such that $w\left(z_{0}\right)=i x, x \in \mathbb{R}$. To prove the required result, it is now sufficient to prove that

$$
\left|(1+\alpha) u\left(z_{0}\right)-\alpha u\left(z_{0}\right)[(1-\delta) i x+\delta]-1\right| \geq M .
$$

By making use of (3), we have

$$
\begin{aligned}
\left|(1+\alpha) u\left(z_{0}\right)-\alpha u\left(z_{0}\right)[(1-\delta) i x+\delta]-1\right| & \geq\left|[1+\alpha(1-\delta)-\alpha(1-\delta) i x] u\left(z_{0}\right)\right|-1 \\
& =\sqrt{[1+\alpha(1-\delta)]^{2}+\alpha^{2}(1-\delta)^{2} x^{2}}\left|u\left(z_{0}\right)\right|-1 \\
& \geq|1+\alpha(1-\delta)|\left|u\left(z_{0}\right)\right|-1 \\
& \geq|1+\alpha(1-\delta)|\left(1-\frac{\beta M}{\beta-\alpha}\right)-1 \geq M .
\end{aligned}
$$

Now (7) is true in view of (1) and therefore, (6) holds. Hence $\Re(w(z))>0$, i.e.,

$$
-\Re\left(\frac{z f^{\prime}(z)}{f(z)}\right)>\delta, 0 \leq \delta<1, z \in \mathbb{E} .
$$

Remark 1. Let $\alpha, \beta, \delta$ be real numbers such that $\alpha<\frac{2}{\delta-1}, 0 \leq \delta<1, \beta>0$ and if $f(z) \in \Sigma$ satisfies

$$
\left|\left(\frac{1}{z f(z)}\right)^{\beta}\left(\frac{1}{\alpha}+1+\frac{z f^{\prime}(z)}{f(z)}\right)-\frac{1}{\alpha}\right|<\frac{(\beta-\alpha)[2+\alpha(1-\delta)]}{\alpha^{2}[1+\beta(1-\delta)]}
$$

then

$$
-\Re\left(\frac{z f^{\prime}(z)}{f(z)}\right)>\delta, z \in \mathbb{E}
$$

Letting $\alpha \rightarrow \infty$ in above remark, we get the following result: 
Theorem 6. Let $\beta, \delta$ be real numbers such that $\beta>0,0 \leq \delta<1$ and let $f(z) \in \Sigma$ satisfy

$$
\left|\left(\frac{1}{z f(z)}\right)^{\beta}\left(1+\frac{z f^{\prime}(z)}{f(z)}\right)\right|<\frac{1-\delta}{1+\beta(1-\delta)}
$$

then

$$
-\Re\left(\frac{z f^{\prime}(z)}{f(z)}\right)>\delta, z \in \mathbb{E}
$$

\section{Deductions}

Setting $\beta=1$ in Theorem 5 , we obtain

Corollary 1. Let $\alpha$ and $\delta$ be real numbers such that $\alpha<\frac{2}{\delta-1}, 0 \leq \delta<1$ and suppose that $f \in \Sigma$ satisfies

$$
\left|\frac{1}{z f(z)}\left(1+\alpha+\alpha \frac{z f^{\prime}(z)}{f(z)}\right)-1\right|<\frac{(1-\alpha)(2+\alpha(1-\delta))}{\alpha(2-\delta)}, z \in \mathbb{E},
$$

then

$$
-\Re\left(\frac{z f^{\prime}(z)}{f(z)}\right)>\delta, z \in \mathbb{E}
$$

i.e., $f \in \mathcal{M S}^{*}(\delta), z \in \mathbb{E}$.

Writing $\delta=0$ in above corollary, we get the following result:

Corollary 2. Let $f \in \Sigma$ satisfy

$$
\left|\frac{1}{z f(z)}\left(1+\alpha+\alpha \frac{z f^{\prime}(z)}{f(z)}\right)-1\right|<\frac{(1-\alpha)(2+\alpha)}{2 \alpha}, z \in \mathbb{E},
$$

then $f \in \mathcal{M S}^{*}, z \in \mathbb{E}$.

Setting $\beta=1$ in Theorem 6, we get the following result:

Corollary 3. Let $\delta$ be a real number such that $0 \leq \delta<1$ and let $f(z) \in \Sigma$ satisfy

$$
\left|\frac{1}{z f(z)}\left(1+\frac{z f^{\prime}(z)}{f(z)}\right)\right|<\frac{1-\delta}{2-\delta}
$$

then

$$
-\Re\left(\frac{z f^{\prime}(z)}{f(z)}\right)>\delta, z \in \mathbb{E} .
$$

Setting $\delta=0$ in above corollary, we get the following result:

Corollary 4. Let $f(z) \in \Sigma$ satisfy

$$
\left|\frac{1}{z f(z)}\left(1+\frac{z f^{\prime}(z)}{f(z)}\right)\right|<\frac{1}{2}
$$

then $f \in \mathcal{M S}^{*}, z \in \mathbb{E}$.

Author Contributions: All authors contributed equally to the writing of this paper. All authors read and approved the final manuscript.

Conflicts of Interest: "The authors declare no conflict of interest." 


\section{References}

[1] Kargar, R., Ebadian, A., \& Sokol, J. (2016). On p-Valently Meromorphic-Strongly Starlike and Convex Functions. International Journal of Analysis and Applications, 12(1), 62-65.

[2] Goswami, P., Bulboacă, T., \& Alqahtani, R. T. (2016). Simple sufficient conditions for starlikeness and convexity for meromorphic functions. Open Mathematics, 14(1), 557-566.

[3] Sahoo, P., Singh, S., \& Zhu, Y. (2011). Some starlikeness conditions for the analytic functions and integral transforms. Journal of Nonlinear Analysis and Application, 2011, 1-10.

[4] Obradovic, M. (1998). A class of univalent functions I. Hokkaido Mathematical Journal, 27(2), 329-335.

[5] Obradovic, M. (1999). A class of univalent functions II. Hokkaido Mathematical Journal, 28(3), 557-562.

[6] Obradovic, M., Ponnusamy, S., Singh, V., \& Vasundhra, P. (2002). Univalency, starlikeness and convexity applied to certain classes of rational functions. Analysis-International Mathematical Journal of Analysis and its Application, 22(3), 225-242.

[7] Hallenbeck, D. J., \& Ruscheweyh, S. (1975). Subordination by convex functions. Proceedings of the American Mathematical Society, 52(1), 191-195. 